\title{
Efecto de la fertilización mineral y de un fertilizante biológico en piña [Ananas comosus (L.) Merr.] en el cultivar MD2 ('Golden')
}

\section{Effect of mineral fertilization and organic fertilization on pineapple [Ananas comosus (L.) Merr.] cultivar MD2 ('Golden')}

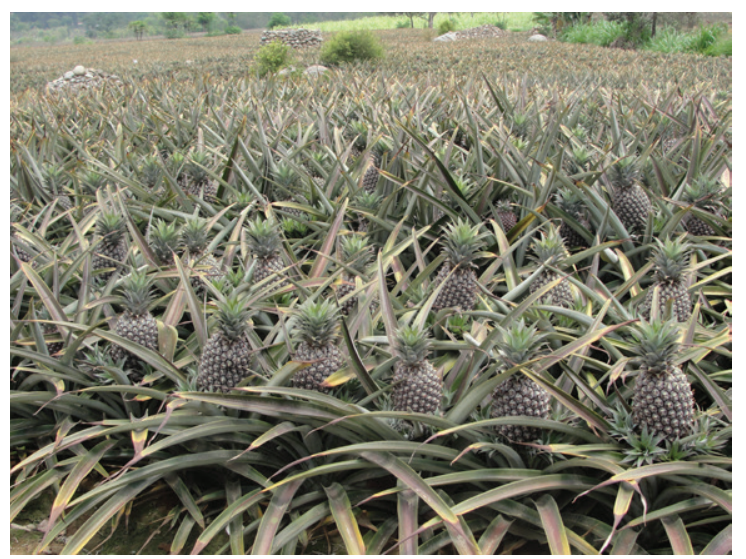

CARLOS MARCA-HUAMANCHA

RICARDO BORJAS-VENTURA', 3

DIANA REBAZA-FERNÁNDEZ²

SEGUNDO BELLO-AMEZ1

ALBERTO JULCA-OTINIANO'

\section{RESUMEN}

En el cultivo de piña en el Perú existe carencia de estudios sobre la fertilización, con fuentes orgánicas e inorgánicas. Por tal motivo, se llevó a cabo este experimento cuyos objetivos fueron determinar la influencia del abono orgánico Alopes Forte, con y sin una fertilización mineral, sobre el crecimiento vegetativo, la productividad, la calidad del fruto y aspectos económicos en la producción de este cultivo. El material vegetal usado fue la piña cultivar 'Golden', la cual fue sometida a los siguientes tratamientos: T1: Testigo; T2: Alopes Forte (25\%); T3: Alopes Forte (50 \%o); T4: Alopes Forte (20 \%o) + N150-P75-K200-Mg25-Ca25; T5: Alopes Forte (20 \%o) + N250-P125-K300-Mg50-Ca50; T6: N300-P150-K400-Mg50-Ca50 y T7: N500-P250-K600-Mg100Ca100. Los resultados muestran que no hubo diferencias entre los tratamientos para el número de hojas, sin embargo en la longitud, peso fresco y seco de la hoja D, se destaca el tratamiento $\mathrm{T} 7$, mientras que en tenor nitrógeno (hoja D) fueron los tratamientos T4, T5, T6 y T7 los que mostraron mejor resultado. Tanto en las variables de calidad del fruto (diámetro inferior, medio, superior, diámetro del eje central del fruto, número de frutillos por fruto, longitud del fruto, peso fresco del fruto con corona y sin corona, y tenor de sólidos solubles) como en el ingreso y rentabilidad en la producción de piña se destacó el tratamiento T7. Estos resultados demuestran que el abono orgánico usado no tuvo influencia sobre las variables evaluadas, además, que el uso de fertilizantes sintéticos afectó positivamente a las variables en estudio.

Palabras clave adicionales: costos, producción, rentabilidad, calidad del fruto, hoja D.

Facultad Agronomía, Departamento Fitotecnia, Grupo Investigación Agricultura y Desarrollo Sustentable en el Trópico Peruano, Universidad Nacional Agraria La Molina, Lima (Perú). ORCID Marca-Huamancha, C.: 0000-00021718-6804; ORCID Borjas-Ventura, R.: 0000-0001-7819-1810; ORCID Bello-Amez, S.: 0000-0002-2405-7138; ORCID Julca-Otiniano, A.: 0000-0002-3433-9032

2 Facultad de Economía y Planificación, Departamento de Estadística e Informática, Universidad Nacional Agraria La Molina, Lima (Perú). ORCID Rebaza-Fernández, D.: 0000-0002-6105-5588

Autor para correspondência.rborjas@lamolina.edu.pe 


\section{ABSTRACT}

In Peruvian pineapple cultivation, there is a lack of fertilizer studies with organic and inorganic sources. For that reason, a study was done in order to determine the influence of Alopes Forte organic fertilizer, with and without mineral fertilization, on some aspects of pineapple cultivation, such as vegetative growth, productivity, fruit quality and economic variables. The pineapple cultivar 'Golden' was used, which was subjected to the following treatments: T1: Control; T2: Alopes Forte (25\%o); T3: Alopes Forte (50\%o); T4: Alopes Forte (20 \%o) + N150-P75K200-Mg25-Ca25; T5: Alopes Forte (20 \%o) + N250-P125-K300-Mg50-Ca50; T6: N300-P150-K400-Mg50-Ca50 and T7: N500-P250-K600-Mg100-Ca100. The results showed that there were no differences between the treatments for the number of leaves; however, treatment T7 had higher values for leaf D length and weight (fresh and dry), while treatments T4, T5, T6 and T7 presented the better result for nitrogen (leaf D). Fruit quality variables (lower, middle and upper diameter, central axis fruit diameter, number of berries per fruit, pineapple length, fruit fresh weight (with and without crown) and content of soluble solids, as well as the pineapple production income and profitability, showed the highest values in T7. These results indicate that the organic fertilizer did not influence on the evaluated variables; on the contrary, the use of synthetic fertilizers had a positive effect.

Additional key words: costs, production, profitability, fruit quality, D leaf.

Fecha de recepción: 06-11-2017 Aprobado para publicación: 30-01-2018

INTRODUCCIÓN

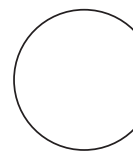

La piña [Ananas comosus (L.) Merr.] es originaria de América tropical y tiene importancia económica en diversos países del mundo. Es consumida como fruta fresca, en jugos, conservas y otros derivados. Según el Ministerio de Agricultura y Riego (2016) el Perú tuvo una producción de 130 mil TM en 15.182 ha. A pesar de la poca área de producción, la piña es una especie muy importante en la economia peruana, principalmente para los pequeños agricultores, ya que es cultivada en regiones con poco desarrollo industrial, tornándose en una fuente de trabajo importante.

El manejo de la fertilización es uno de los factores de mayor relevancia en el cultivo de piña. Una fertilización equilibrada asegura una buena producción, y consecuentemente una alta rentabilidad para los agricultores que dependen de este cultivo. A pesar de estos benefícios, en Perú, hay pocos trabajos que fueron realizados sobre dicho tema (Bello et al., 2015). Por otro lado, el desconocimiento de las dosis ideales de fertilización, en función del tipo de suelo donde es plantada esta especie, puede acarrear grandes perjuicios para los productores, pues el exceso o falta de nutrientes, puede causar una baja en la productividad, aumentar los costos de producción, bajar la calidad de los frutos y contaminar los suelos y aguas. Estos perjuicios no solo pueden afectar la economía del pequeño agricultor sino también al propio agroecosistema (Almaguer, 2013).

Una de las formas de disminuir los posibles perjuicios económicos y ambientales, causados por la mala utilización de fertilizantes sintéticos, es el empleo de forma simultánea de fuentes sintéticas y orgánicas, de forma que se tenga un menor uso de las primeras. Diversos trabajos verificaron las ventajas de la utilización de fertilizantes orgánicos sobre el desarrollo de diferentes especies (Aseri et al., 2008; Mujica et al., 2014). Así también, el uso conjunto de abonos orgánicos y sintéticos, viene siendo usados cada vez más en otros cultivos de importancia económica como el banano (Ramos et al., 2016) y la papa (Luna et al., 2016).

Actualmente, en el Perú, existen diversos fertilizantes orgánicos que precisan ser probados bajo diferentes condiciones agroecológicas, para determinar el efecto de ellos sobre el crecimiento y desarrollo de plantas. Entre estos fertilizantes, presentes en el mercado peruano, se destaca Alopes Forte (de la empresa Noga-Fer Perú), el cual es producido por la fermentación de subproductos de la indústria hidrobiológica y que además presenta certificación para uso en la agricultura orgánica. 
En este contexto de incertidumbre, el trabajo tiene como objetivo general evaluar las respuesta de Ananas comosus, cultivar MD2 ('Golden'), a la fertilización orgánica, mineral y a la aplicación conjunta de estas formas de fertilización. Asimismo, los objetivos específicos fueron la evaluación del efecto de las fuentes de abono sobre variables vegetativas, productivas y económicas en la producción de piña.

\section{MATERIALES Y MÉTODOS}

El experimento fue realizado desde noviembre del 2011 hasta marzo del 2013, en el fundo Santa Teresa, localizado en el município de Río Negro, Satipo Junín (Perú), cuyas coordenadas geográficas son $11^{\circ} 02^{\prime} 00^{\prime \prime}$ S y 7402'00" W, con una altitud de $850 \mathrm{msnm}$. El ensayo tuvo una duración de 16 meses donde se evaluó un ciclo de producción. En la figura 1 se encuentran los datos mensuales de temperatura y precipitación pluvial durante el período experimental.

Antes de la instalación del experimento, se realizó el análisis de los primeiros $30 \mathrm{~cm}$ de suelo, obteniendose: $\mathrm{pH}$ de 5,32 (determinado en agua, en relación suelo-agua 1:1), un tenor de materia orgánica de 3,14\%, $6,90 \mathrm{mg} \mathrm{kg}^{-1}$ de $\mathrm{P}$ disponible, $183 \mathrm{mg} \mathrm{kg}^{-1}$ de $\mathrm{K}$ disponible, 9,28 $\mathrm{cmol}_{(+)} \mathrm{kg}^{-1}$ de CIC y un porcentaje de saturación de bases de $75,65 \%$. Respecto al cálcio y al magnésio, el suelo tuvo 5,82 y $0,33 \mathrm{cmol}_{(+)} \mathrm{kg}^{-1}$ de estos nutrientes respectivamente (Soil Science Society of America, 1982). El análisis físico mostró que el suelo del experimento se encuentra dentro de la clase textural franco-arenoso.

Se usó material vegetal del cultivar MD2 ('Golden'), obtenido de la cosecha de piña anterior en el fundo
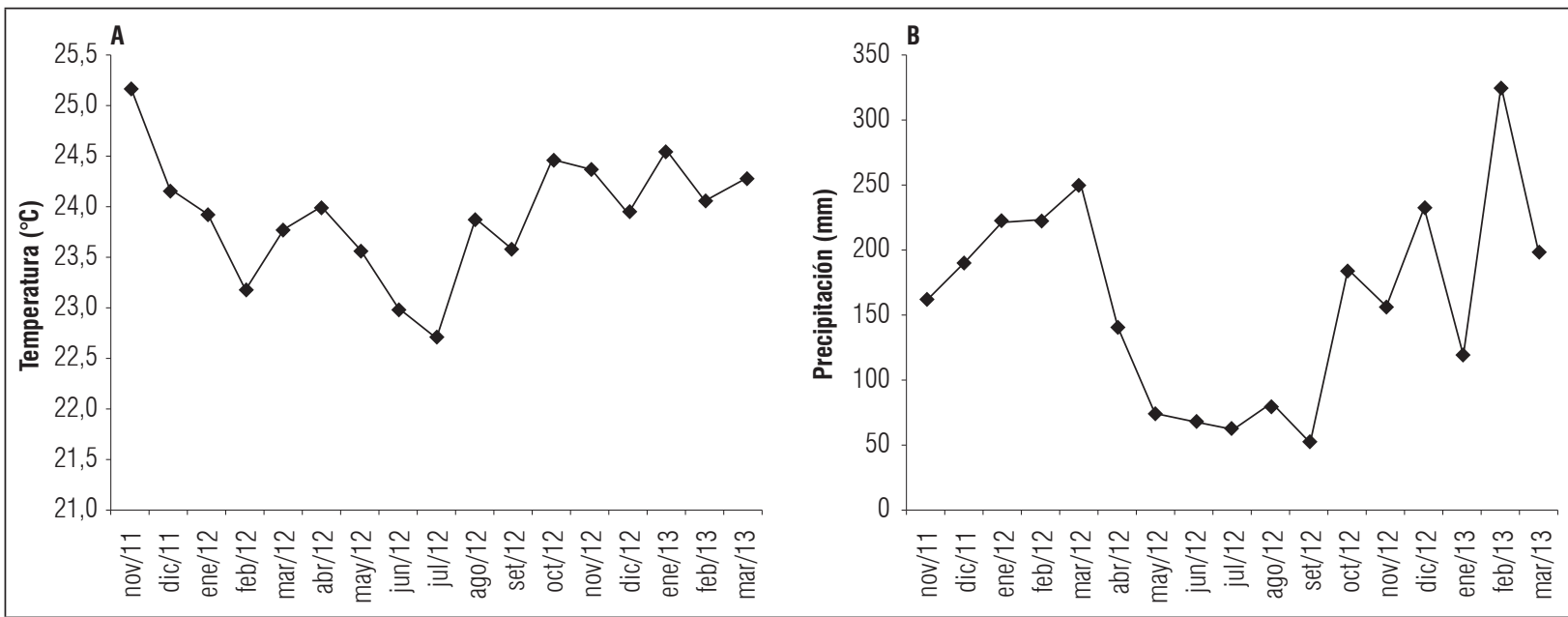

Figura 1. Temperatura (A) y precipitación (B) durante el ensayo de piña. Datos de la estación meteorológica del fundo Santa Teresa.

Tabla 1. Tratamientos de los fertilizantes sintéticos y orgánico aplicados durante el ensayo de piña.

\begin{tabular}{|c|c|c|c|c|c|c|}
\hline \multirow{2}{*}{ Tratamientos } & \multirow{5}{*}{ Fertilizante orgánico } & \multicolumn{5}{|c|}{ Fertilizante sintético $\left(\mathrm{kg} \mathrm{ha}{ }^{-1}\right)$} \\
\cline { 3 - 7 } & 0 & $\mathrm{~N}$ & $\mathrm{P}_{2} \mathrm{O}_{5}$ & $\mathrm{~K}_{2} \mathrm{O}$ & $\mathrm{Mg0}$ & $\mathrm{Ca0}$ \\
\hline T1 & 0 & 0 & 0 & 0 & 0 \\
\hline T2 & Alopes Forte (25\%) & 0 & 0 & 0 & 0 & 0 \\
\hline T3 & Alopes Forte (50\%) & 0 & 0 & 0 & 25 & 25 \\
\hline T4 & Alopes Forte (20\%) & 150 & 75 & 200 & 50 & 50 \\
\hline T5 & Alopes Forte (20\%) & 250 & 125 & 300 & 50 & 50 \\
\hline T6 & 0 & 300 & 150 & 400 & 100 & 100 \\
\hline T7 & 0 & 500 & 250 & 600 & 5 & 0 \\
\hline
\end{tabular}


Santa Teresa. El material vegetal consistió en hijuelos de plantas con un promedio de $250 \mathrm{~g}$. Antes de ser plantado, todo el material de propagación fue sometido a tratamientos fitosanitarios (fosetil aluminio) a fin de evitar la entrada de patógenos.

La preparación del suelo fue de tipo convencional, siendo incorporado los resíduos vegetales de la cosecha anterior (tanto malezas como los restos de piña). Después de la preparación del suelo, se inició la siembra (noviembre 2011) del material vegetal. Para aumentar la densidad de plantas se usó la siembra en doble hilera con un distanciamiento entre líneas de $0,3 \mathrm{~m}$ y entre plantas de $0,4 \mathrm{~m}$, con un ancho de cama de 1,4 m. Cada hilera tuvo nueve plantas, dando una densidade total de 48.000 plantas/ha.

El control de plagas y enfermedades (Phytophthora spp. y Dysmicoccus brevipes, además de Thecla basilides) fue realizado con Mancozeb + Metalaxil y Clorpyrifos, en este caso la aplicación de estos plaguicidas fue realizado en todos los tratamientos de forma homogénea. El control de malezas fue realizado manualmente.

La inducción floral (IF) se realizó 10 meses después de la siembra (agosto 2012), tiempo que coincidió con la llegada de las lluvias. Para la IF se usó Etefon (48\%) a una dosis de $0,15 \%$. A los $100 \mathrm{~d}$ de la IF, los frutos fueron cubiertos con bolsas plásticas para el control de Melanoloma viatrix. En marzo del 2013 se realizó la cosecha de frutos (16 meses después de la siembra).

El diseño usado en el experimento fue el de bloques al azar con siete tratamientos (Tab. 1) y cuatro repeticiones. Por tanto, se tuvo 28 unidades experimentales. Cada unidad experimental estuvo formada por 54 plantas. La toma de muestra fue hecha en las plantas centrales de cada unidad experimental.

El nitrógeno $(\mathrm{N})$, magnesio $(\mathrm{Mg})$, calcio $(\mathrm{Ca})$ y potasio (K) fueron divididos en cuatro partes iguales, mientras que el fósforo $(\mathrm{P})$ en su totalidad fue aplicado durante la siembra. Las fuentes utilizadas fueron fosfato diamónico ( $18 \%$ de $\mathrm{N}$ y $46 \%$ de $\mathrm{P}_{2} \mathrm{O}_{5}$ ), urea ( $46 \%$ de $\mathrm{N}$ ), cloruro de potasio $\left(60 \%\right.$ de $\left.\mathrm{K}_{2} \mathrm{O}\right)$, sulfato de magnesio y potasio ( $22 \%$ de $\mathrm{K}_{2} \mathrm{O}$ y $18 \%$ de $\mathrm{MgO}$ ) y nitrato de calcio ( $15 \%$ de $\mathrm{N}$ y $26 \%$ de $\mathrm{CaO}$ ). En los tratamientos con Alopes Forte (Tab. 2) se usó las siguientes dosis: $20 \%$, $25 \%$ y $50 \%$. La dosis de $2 \%$ fue aplicado solo una vez en fondo de surco $1 \mathrm{~d}$ antes de la siembra y con adición de abono sintético. Las dosis de 25 y $50 \%$ o fueron aplicados sin adición de fertilizantes minerales en cinco partes iguales de 5 y $10 \%$, respectivamente, a los 0, 45, 90, 135 y 180 días después de la siembra (dds) y antes de la inducción floral (descrito anteriormente).

\section{Tabla 2. Característica química de Alopes-Forte ${ }^{1}$.}

Característica Concentración Características Concentración

\begin{tabular}{|l|c|l|c|}
\hline $\mathrm{pH}$ & 4,0 & Magnesio & $384,00 \mathrm{mg} \mathrm{L}^{-1}$ \\
\hline $\begin{array}{l}\text { Materia } \\
\text { orgánica }\end{array}$ & $311,00 \mathrm{~g} \mathrm{~L}^{-1}$ & Hierro & $32,40 \mathrm{mg} \mathrm{L}^{-1}$ \\
\hline Nitrógeno & $22,50 \mathrm{~g} \mathrm{~L}^{-1}$ & Cobre & $0,80 \mathrm{mg} \mathrm{L}^{-1}$ \\
\hline Fósforo & $2,10 \mathrm{~g} \mathrm{~L}^{-1}$ & Zinc & $3,10 \mathrm{mg} \mathrm{L}^{-1}$ \\
\hline Potasio & $3,70 \mathrm{~g} \mathrm{~L}^{-1}$ & Manganeso & $1,20 \mathrm{mg} \mathrm{L}^{-1}$ \\
\hline Cálcio & $1,40 \mathrm{~g} \mathrm{~L}^{-1}$ & Boro & $250,80 \mathrm{mg} \mathrm{L}^{-1}$ \\
\hline
\end{tabular}

${ }^{1}$ Recomendación de la empresa Noga-Fer, Lima, Perú.

Según los datos proporcionados por el fabricante (Noga-Fer, Lima, Perú), Alopes Forte es un fertilizante orgánico que puede ser usado tanto en la agricultura orgánica como convencional. Entre las principales características del producto se encuentra la capacidad de estimular la actividad microbiana del suelo.

En la fase vegetativa, a los 90,135 y 180 dds, se evaluó el número de hojas, la longitud, peso fresco, peso seco y concentración de nitrógeno en la hoja D (método Kjeldahl) (Guitián y Carballas, 1976). Se evaluaron estas características de la hoja $\mathrm{D}$, puesto que ella se encuentra fuertemente relacionada con el crecimiento del fruto.

Una vez cosechado el fruto (16 meses después de la siembra), se midió el diámetro inferior, medio y superior, para esto se usó una regla milimetrada y se cortó el fruto en la parte central, superior e inferior, siendo en esos cortes donde se midieron los diámetros. El diámetro del eje central del fruto también fue medido con una regla en la parte central del fruto. Además, fueron evaluados el número de frutillos ("ojos" del fruto), para lo cual se contaron el número de frutillos por piña. También, se cuantificó la longitud de cada fruto (que tuvo como base la parte inferior y llegó hasta la parte superior del mismo), el tenor de sólidos solubles totales (con el uso de un Brixómetro), asi como el peso de frutos con y sin corona (Bello et al., 2015).

Se realizó una clasificación de frutos, en cinco calibres diferentes en función del peso, los cuales fueron codificados como C6, C8, C10, C12 y C14 cada uno 
correspondiente a 2,5-3,2 kg; 2,1-2,5 kg; 1,7-2,1 kg; 1,4-1,7 kg; 1,2-1,4 kg, respectivamente. Se determinó la productividad, contando y pesando el número de frutos totales en cada tratamiento (llevándolo todo a hectárea). Para determinar el ingreso bruto, se cuantificó el número y el peso de frutos/calibre (5), luego se multiplicó por el precio de cada tipo de piña (calibre) para cada tratamiento. Asimismo, se anotó la utilidad, siendo esta la diferencia entre el ingreso bruto y los costos totales, y finalmente se evaluó la rentabilidad (\%), dividiendo la utilidad entre el costo total y multiplicando el resultado por 100. Es importante resaltar que, a pesar que la moneda nacional peruana es el Sol (S/.), los valores monetarios, en este ensayo, están presentados en US\$, siendo el tipo de cambio: US $\$ 1=S / 3,20$. Este tipo de cambio se tomó de la Superintentendia Nacional de Administración Tributaria (SUNAT).

Los datos de las variables evaluadas fueron sometidas al análisis de variancia (ANOVA) y las medias fueron comparadas por el test de Tukey $(P \leq 0,05)$, asimismo, se empleó el software estadístico AGROSTAT (Barbosa y Junior, 2010).

\section{RESULTADOS Y DISCUSIÓN}

De acuerdo a los resultados obtenidos, se observa que el número de hojas entre los 90 y 180 dds no presentó diferencia significativa entre las medias $(P \leq 0,05)$. Por otra parte, a los 135 dds se notó mayor número de hojas en los tratamientos T6 y T7. De forma general se notó un aumento de las cantidades de hojas, entre la primera y la última evaluación, de más del 70\% (Tab. 3).

Es importante mencionar que solo en la segunda evaluación se encontró diferencia estadística, siendo los mayores valores para los tratamientos con mayor cantidad de abono sintético (T6 y T7), especialmente nitrógeno $(\mathrm{N})$. En este sentido, Omotoso y Akinrinde (2013) reportaron que a mayor cantidad de N, mayor cantidad de hojas en piña se formaron.

Asimismo, estos resultados indicarían que a los 180 dds la planta comienza a prepararse para pasar a la etapa productiva (cabe resaltar que a los 10 meses después de la siembra se inició la inducción floral), siendo innecesario aumentar el número de hojas, teniendo mayor importancia la acumulación de mayores cantidades de nutrientes para que sean traslocados al fruto, lo que podría explicar la ausencia de diferencias en la última evaluación.

La falta de emisión de hojas, antes de pasar a la etapa productiva, es un fenómeno que fue observado por Bello et al. (2015) en piña 'Cayena Lisa' en condiciones agroecológicas semejantes. La hoja D tiene importancia práctica en el manejo de la piña, ya que la longitud

\section{Tabla 3. Número de hojas y longitud de hoja D de piña, cultivar MD2 ('Golden'), en tres épocas de evaluación, en función de la aplicación de abono sintético y con el fertilizante biológico.}

\begin{tabular}{|l|c|c|c|c|c|c|}
\hline \multirow{2}{*}{ Tratamientos } & \multicolumn{3}{|c|}{ Número de hojas } & \multicolumn{3}{c|}{ Longitud de hoja D (cm) } \\
\cline { 2 - 7 } & $\begin{array}{c}90 \\
\text { dds }\end{array}$ & $\begin{array}{c}135 \\
\text { dds }\end{array}$ & $\begin{array}{c}180 \\
\text { dds }\end{array}$ & $\begin{array}{c}90 \\
\text { dds }\end{array}$ & $\begin{array}{c}135 \\
\text { dds }\end{array}$ & $\begin{array}{c}180 \\
\text { dds }\end{array}$ \\
\hline T1 & $3,75 a$ & $3,73 c$ & $6,63 a$ & $45,19 \mathrm{~d}$ & $40,76 \mathrm{c}$ & $58,64 \mathrm{~d}$ \\
\hline T2 & $4,05 \mathrm{a}$ & $4,05 \mathrm{bc}$ & $6,90 \mathrm{a}$ & $49,64 \mathrm{bcd}$ & $55,88 \mathrm{bc}$ & $63,62 \mathrm{~cd}$ \\
\hline T3 & $3,98 \mathrm{a}$ & $3,83 \mathrm{c}$ & $6,78 \mathrm{a}$ & $46,85 \mathrm{~cd}$ & $54,78 \mathrm{bc}$ & $62,00 \mathrm{~d}$ \\
\hline T4 & $4,50 \mathrm{a}$ & $4,33 \mathrm{abc}$ & $7,50 \mathrm{a}$ & $49,89 \mathrm{bcd}$ & $57,65 \mathrm{bc}$ & $67,23 \mathrm{bc}$ \\
\hline T5 & $4,40 \mathrm{a}$ & $4,10 \mathrm{bc}$ & $7,60 \mathrm{a}$ & $54,69 \mathrm{ab}$ & $62,43 \mathrm{ab}$ & $70,25 \mathrm{~b}$ \\
\hline T6 & $4,28 \mathrm{a}$ & $4,65 \mathrm{ab}$ & $7,53 \mathrm{a}$ & $52,70 \mathrm{abc}$ & $57,80 \mathrm{bc}$ & $69,06 \mathrm{~b}$ \\
\hline T7 & $4,33 \mathrm{a}$ & $4,80 \mathrm{a}$ & $7,50 \mathrm{a}$ & $57,00 \mathrm{a}$ & $68,00 \mathrm{a}$ & $76,57 \mathrm{a}$ \\
\hline CV & 9,11 & 6,16 & 6,90 & 5,40 & 7,13 & 3,30 \\
\hline EE & 0,19 & 0,13 & 0,25 & 1,40 & 2,07 & 1,10 \\
\hline
\end{tabular}

T1: Testigo; T2: Alopes Forte 25\%; T3: Alopes Forte 50\%; T4: Alopes Forte 20\%o + 150N-75 $\mathrm{P}_{2} \mathrm{O}_{5}-200 \mathrm{~K}_{2} \mathrm{O}-25 \mathrm{MgO}-25 \mathrm{CaO}$; T5: Alopes Forte 20\%o $+250 \mathrm{~N}-125 \mathrm{P}_{2} \mathrm{O}_{5}$ -

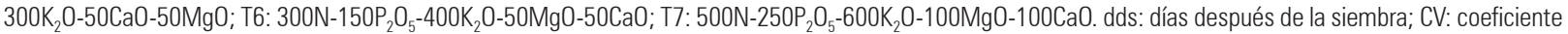
de variación; EE: error estándar.

Promedios con letras distintas, en la misma columna, indican diferencia significativa según la prueba de Tukey $(P \leq 0,05)$. 
de ella está fuertemente relacionada con el crecimiento del fruto (Lacoeuilhe, 1987). Así también, esta variable puede mostrar indícios de alguna diferencia en el manejo agronómico y nutricional de este cultivo (Rodríguez et al., 2016; Guarçoni y Ventura, 2011). En este experimento, con el paso del tiempo, se notó un aumento longitudinal de la hoja D con $76.57 \mathrm{~cm}$ a los $180 \mathrm{dds}$, mientras que los menores valores fueron observados en los tratamentos T1, T2 y T3 con 58,$64 ; 63,62$ y $62,00 \mathrm{~cm}$, respectivamente $(P \leq 0,05)$ (Tab. 2). Cardoso et al. (2013), evaluando dos fuentes nitrogenadas en la dinámica de crecimiento de la hoja $\mathrm{D}$, relataron que la fuente sintética tuvo efectos significativamente superior a la fuente orgánica, semejante a lo notado en este trabajo.

Por otra parte, Vilela et al. (2015) relataron que tanto el peso seco como fresco de la hoja $\mathrm{D}$, tienen una correlación de 82 y $84 \%$ con el crecimiento del fruto, respectivamente. En todos los momentos de evaluación, el peso seco y fresco de la hoja D fueron superiores en el tratamiento $\mathrm{T} 7$, el cual tuvo las mayores dosis de fertilizante sintético (Tab. 4). Asimismo, el menor efecto del fertilizante orgánico observado en este experimento, podría explicarse por la baja tasa de mineralización de la materia orgánica contenida en este produto. En otro ensayo con 'Golden', se encontró una relación directa entre el aumento de la dosis de fertilizantes sintéticos y el aumento del peso seco de la hoja D (Guarçoni y Ventura, 2011).
Los tenores de nitrógeno en la hoja D mostraron una tendencia de aumento en los tratamentos T5, T6 y $\mathrm{T} 7$, todos con aplicación de abono sintético $(P \leq 0,05)$. Este hecho se debe, posiblemente, a que los nutrientes contenidos en los fertilizantes sintéticos estaban disponibles con mayor rapidez para ser utilizados por las plantas. Para el caso del N, la mineralización de este nutriente hasta alcanzar un tenor de $100 \mathrm{mg} \mathrm{kg}^{-1}$ de suelo, puede llevar hasta $270 \mathrm{~d}$, estando influenciado por las condiciones climáticas y los tipos de abonos usados (Carneiro et al., 2013).

Dentro de los macronutrientes, el $\mathrm{N}$ es de suma importancia, ya que podemos encontrarlo formando parte de biomoléculas importantes como la clorofila (Lacoeuilhe, 1987; Schlemmer et al., 2013), la cual se encuentra directamente relacionada con la fotosíntesis, que, a su vez, influencia el aumento de peso seco. En este sentido, Caetano et al. (2013) y Bello et al. (2015) demostraron que con el aumento en las dosis de $\mathrm{N}$, hubo un incremento en el peso seco de la hoja $\mathrm{D}$ en los cultivares 'Vitoria' y 'Cayena Lisa'. En este trabajo de forma general, en los tratamientos con mayor materia seca, se detectó también mayores tenores de $\mathrm{N}$ en la hoja $\mathrm{D}$.

La evaluación del diámetro de fruto es importante pues la agroindústria tiene preferencia por aquellos de forma cilíndrica, ya que permiten obtener mayor rendimento agroindustrial (Bello et al., 2015).

Tabla 4. Peso fresco y peso seco de la hoja D de piña, cultivar MD2 ('Golden'), en tres momentos de evaluación, en función de la aplicación de abono sintético y el fertilizante orgánico. Tenor de nitrógeno en la hoja D.

\begin{tabular}{|l|c|c|c|c|c|c|c|}
\hline \multirow{2}{*}{ Tratamientos } & \multicolumn{3}{|c|}{ Peso fresco (g) } & \multicolumn{3}{c|}{ Peso seco (g) } & N \\
\cline { 2 - 8 } & $\begin{array}{c}90 \\
\text { dds }\end{array}$ & $\begin{array}{c}135 \\
\text { dds }\end{array}$ & $\begin{array}{c}180 \\
\text { dds }\end{array}$ & $\begin{array}{c}90 \\
\text { dds }\end{array}$ & $\begin{array}{c}135 \\
\text { dds }\end{array}$ & $\begin{array}{c}180 \\
\text { dds }\end{array}$ & 20 \\
\hline T1 & $15,17 \mathrm{c}$ & $20,68 \mathrm{c}$ & $31,00 \mathrm{e}$ & $1,66 \mathrm{c}$ & $2,32 \mathrm{c}$ & $3,07 \mathrm{~d}$ & $1,60 \mathrm{abc}$ \\
\hline T2 & $18,66 \mathrm{bc}$ & $29,00 \mathrm{~b}$ & $38,30 \mathrm{cde}$ & $2,15 \mathrm{abc}$ & $3,12 \mathrm{~b}$ & $3,61 \mathrm{~cd}$ & $1,50 \mathrm{bc}$ \\
\hline T3 & $18,66 \mathrm{bc}$ & $29,00 \mathrm{~b}$ & $35,35 \mathrm{de}$ & $1,83 \mathrm{bc}$ & $3,14 \mathrm{~b}$ & $4,00 \mathrm{bc}$ & $1,44 \mathrm{c}$ \\
\hline T4 & $20,60 \mathrm{~b}$ & $33,04 \mathrm{~b}$ & $42,97 \mathrm{bcd}$ & $2,28 \mathrm{ab}$ & $3,90 \mathrm{a}$ & $4,32 \mathrm{bc}$ & $1,68 \mathrm{abc}$ \\
\hline T5 & $20,60 \mathrm{~b}$ & $34,60 \mathrm{~b}$ & $47,70 \mathrm{~b}$ & $2,23 \mathrm{ab}$ & $2,98 \mathrm{~b}$ & $4,58 \mathrm{ab}$ & $1,80 \mathrm{a}$ \\
\hline T6 & $19,20 \mathrm{bc}$ & $32,23 \mathrm{~b}$ & $45,49 \mathrm{bc}$ & $2,12 \mathrm{abc}$ & $3,15 \mathrm{~b}$ & $4,01 \mathrm{bc}$ & $1,71 \mathrm{ab}$ \\
\hline T7 & $25,29 \mathrm{a}$ & $42,40 \mathrm{a}$ & $56,00 \mathrm{a}$ & $2,49 \mathrm{a}$ & $4,10 \mathrm{a}$ & $5,12 \mathrm{a}$ & $1,78 \mathrm{a}$ \\
\hline CV & 9,90 & 9,60 & 7,80 & 11,50 & 0,86 & 0,80 & 6,60 \\
\hline EE & 0,97 & 1,51 & 1,70 & 1,20 & 0,14 & 0,17 & 0,05 \\
\hline
\end{tabular}

T1: Testigo; T2: Alopes Forte 25\%;; T3: Alopes Forte 50\%; T4: Alopes Forte 20\%o + 150N-75 $\mathrm{P}_{2} \mathrm{O}_{5}-200 \mathrm{~K}_{2} \mathrm{O}-25 \mathrm{MgO}-25 \mathrm{CaO}$; T5: Alopes Forte 20\%o $+250 \mathrm{~N}-125 \mathrm{P}_{2} \mathrm{O}_{5}$ -

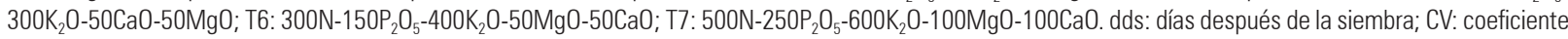
de variación; EE: error estándar.

Promedios con letras distintas, en la misma columna, indican diferencia significativa según la prueba de Tukey $(P \leq 0,05)$. 
Tabla 5. Diámetro inferior (DI), medio (DM), superior (DS) y diámetro del eje central (DEC) del fruto, longitud del fruto (LF),
número de frutillos por fruto (NFF), peso fresco del fruto con corona (PFCC) y sin corona (PFSC) y tenor de sólidos so-
lubles (TSS) de piña, cultivar MD2 ('Golden'), en función de la aplicación de abono sintético y del fertilizante orgánico.

\begin{tabular}{|c|c|c|c|c|c|c|c|c|c|}
\hline \multirow{2}{*}{ Tratamientos } & DI & DM & DS & DEC & $\mathrm{LF}$ & \multirow{2}{*}{$\begin{array}{c}\text { NFF } \\
-\end{array}$} & PFCC & PFSC & \multirow{2}{*}{$\frac{\text { TSS }}{{ }^{\circ} \text { Brix }}$} \\
\hline & \multicolumn{5}{|c|}{$\mathrm{cm}$} & & \multicolumn{2}{|c|}{$\mathrm{kg}$} & \\
\hline $\mathrm{T} 1$ & $10,12 c^{1}$ & $11,17 f$ & $10,28 d$ & $2,64 \mathrm{c}$ & $11,53 \mathrm{e}$ & $77,16 \mathrm{e}$ & $1,23 d$ & $1,10 \mathrm{c}$ & $12,90 \mathrm{e}$ \\
\hline $\mathrm{T} 2$ & $10,29 \mathrm{c}$ & $11,25 f$ & $10,26 d$ & $2,51 \mathrm{~d}$ & 11,93 de & 79,18 e & $1,24 d$ & $1,10 \mathrm{c}$ & 13,02 de \\
\hline T3 & $10,26 c$ & $11,48 \mathrm{e}$ & $9,83 \mathrm{e}$ & $2,56 \mathrm{~cd}$ & $12,30 \mathrm{~d}$ & $83,95 d$ & $1,22 \mathrm{~d}$ & $1,10 \mathrm{c}$ & $13,46 \mathrm{bcd}$ \\
\hline $\mathrm{T} 4$ & $10,26 \mathrm{c}$ & $11,91 \mathrm{~d}$ & $10,12 d$ & $2,24 \mathrm{e}$ & $13,50 \mathrm{c}$ & $89,63 \mathrm{c}$ & $1,50 \mathrm{c}$ & $1,40 \mathrm{~b}$ & $13,57 \mathrm{bc}$ \\
\hline T5 & $10,81 \mathrm{~b}$ & $12,27 \mathrm{c}$ & 10,92 b & $2,78 \mathrm{~b}$ & $13,21 \mathrm{c}$ & $85,68 \mathrm{~cd}$ & $1,61 \mathrm{~b}$ & $1,50 \mathrm{~b}$ & $13,89 \mathrm{~b}$ \\
\hline T6 & $10,96 \mathrm{~b}$ & $12,42 b$ & $10,74 \mathrm{~b}$ & $2,55 \mathrm{~cd}$ & $14,28 \mathrm{~b}$ & $95,52 b$ & $1,63 \mathrm{~b}$ & $1,50 \mathrm{~b}$ & $13,41 \mathrm{~cd}$ \\
\hline $\mathrm{T7}$ & $11,90 \mathrm{a}$ & 12,78 a & 11,61 a & $3,01 \mathrm{a}$ & $15,84 a$ & $103,25 a$ & $1,81 \mathrm{a}$ & $1,65 \mathrm{a}$ & $15,10 \mathrm{a}$ \\
\hline $\mathrm{CV}^{2}$ & 0,81 & 0,39 & 0,67 & 1,54 & 2,30 & 2,21 & 2,84 & 3,41 & 1,50 \\
\hline EE & 0,04 & 0,02 & 0,04 & 0,02 & 0,15 & 0,97 & 0,02 & 0,02 & 0,02 \\
\hline
\end{tabular}

T1: Testigo; T2: Alopes Forte 25\%; T3: Alopes Forte 50\%; T4: Alopes Forte 20\%o + 150N-75 $\mathrm{P}_{2} \mathrm{O}_{5}-200 \mathrm{~K}_{2} \mathrm{O}-25 \mathrm{MgO}-25 \mathrm{CaO}$; T5: Alopes Forte 20\%o $+250 \mathrm{~N}-125 \mathrm{P}_{2} \mathrm{O}_{5}$ -

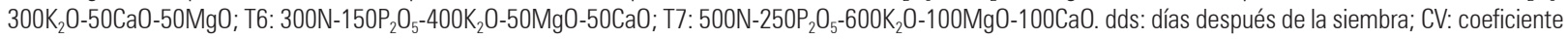
de variación; EE: error estándar.

Promedios con letras distintas, en la misma columna, indican diferencia significativa según la prueba de Tukey $(P \leq 0,05)$.

De acuerdo con la tabla 5, para el diámetro inferior, medio, superior y diámetro del eje central del fruto, el tratamiento T7 fue superior a los otros tratamientos. Por otro lado, los tratamientos T1, T2 y T3 mostraron menores valores. Los resultados obtenidos se deben, probablemente, a la mayor disponibilidad de nutrientes por los tratamentos aplicados (fertilizante sintéti$\mathrm{co}$ ), que a su vez, fueron aprovechados por las plantas para el crecimiento del fruto.

En investigaciones hechas con N, Bello et al. (2015), Caetano et al. (2013) y Omotoso y Akinrinde (2013) verificaron que a medida que aumentaba la cantidad de $\mathrm{N}$ aplicado, también se aumentaba el diámetro de fruto de piña. Efectos parecidos para el potasio (K) fueron relatados por Annisa et al. (2013).

Para el número de frutillos por fruto (NFF), longitud del fruto (LF), peso de fruto con corona (PFCC) y peso del fruto sin corona (PFSC), se observó que el tratamiento T7 fue superior a los demás (Tab. 4). Por otro lado, los tratamientos T1, T2 y T3 tuvieron los menores valores. Los resultados obtenidos, se deben probablemente al mejor aprovechamiento de los nutrientes durante el crecimiento vegetativo de las plantas, lo que a su vez reflejó un mayor crecimiento de fruto. De acuerdo con Pegoraro et al. (2014), la piña exporta nutrientes, durante el desarrollo del fruto, en el siguiente orden: $\mathrm{K}>\mathrm{N}>\mathrm{S}>\mathrm{Ca}>\mathrm{P}>\mathrm{Mg}$, los cuales son fundamentales en el desarrollo cuantitativo y cualitativo del mismo. Resultados similares fueron encontrados por Guarçoni y Aires (2011) en piña 'Golden'.

En relación al tenor de sólidos solubles totales (SST), se verificó que el tratamiento T7 fue superior a los demás tratamientos con aproximadamente 15,1 ${ }^{\circ}$ Brix. Caetano et al. (2013) y Oliveira et al. (2015) informaron que con el aumento de $\mathrm{N}$ y $\mathrm{K}$, también hubo incrementos en los SST.

Para la venta de piña en el mercado peruano, es necesario que el fruto este clasificado, ya que el precio que recibe el agricultor estará en función del tamaño de este. En cuanto al porcentaje de fruto comercializable, se notó que el mayor porcentaje de fruto no comercializable fue obtenido por los tratamientos T2 y T3, mientras el T7 tuvo mayor percentaje de frutos con peso fresco entre 1,7 y 2,1 kg. Se notó que a medida que se incrementaba la dosis de fertilizantes, también aumentaba la productividad (Tab. 6). Omotoso y Akinrinde (2013) y Spironello et al. (2004) verificaron que la aplicación de mayor cantidad de abono mineral, promovió el aumento en la productividad de piña. Además, constataron mayor rentabilidad por hectárea a medida que aumentaba la dosis de abono aplicado, lo que demuestra la importancia de un buen manejo de la fertilización de suelo, que a su vez se puede ver reflejado en el aumento de ingresos, lo que de hecho sucedió en este trabajo, notandose un incremento del 199\% en el ingreso bruto entre el tratamiento T1 y T7 (Tab. 6). 
Tabla 6. Clasificación de frutos, produtividad e ingreso bruto de piña, cultivar MD2 ('Golden'), en función de la aplicación de abono sintético y fertilizante orgánico.

\begin{tabular}{|c|c|c|c|c|c|c|c|c|}
\hline \multirow{3}{*}{ Tratamientos } & \multicolumn{6}{|c|}{ Clasificación de frutos } & \multirow{3}{*}{$\begin{array}{l}\text { Productividad } \\
\mathrm{kg} \mathrm{ha}^{-1}\end{array}$} & \multirow{2}{*}{$\begin{array}{c}\text { Ingreso } \\
\text { bruto }\end{array}$} \\
\hline & C-6 & C-8 & C-10 & C-12 & C-14 & Descarte & & \\
\hline & \multicolumn{6}{|c|}{$\%$} & & US\$ \\
\hline T1 & 0 & 0 & 0 & 10,00 & 45,00 & 45,00 & 59.280 & $8.134,82$ \\
\hline $\mathrm{T} 2$ & 0 & 0 & 2,50 & 5,00 & 45,00 & 47,50 & 59.160 & $8.132,95$ \\
\hline T3 & 0 & 0 & 0 & 2,50 & 52,50 & 45,00 & 58.380 & $7.556,25$ \\
\hline T4 & 0 & 0 & 5,00 & 67,50 & 20,00 & 7,50 & 71.220 & $14.681,52$ \\
\hline T5 & 0 & 0 & 30,00 & 57,50 & 12,50 & 0 & 77.940 & $18.775,72$ \\
\hline T6 & 0 & 0 & 35,00 & 60,00 & 5 & 0 & 79.680 & $19.894,28$ \\
\hline $\mathrm{T7}$ & 0 & 5.00 & 60,00 & 35,00 & 0 & 0 & 86.280 & $24.330,00$ \\
\hline
\end{tabular}

T1: Testigo; T2: Alopes Forte 25\%; T3: Alopes Forte 50\%; T4: Alopes Forte 20\%o + 150N-75 $\mathrm{P}_{2} \mathrm{O}_{5}-200 \mathrm{~K}_{2} \mathrm{O}-25 \mathrm{MgO}-25 \mathrm{CaO}$; T5: Alopes Forte 20\%o $+250 \mathrm{~N}-125 \mathrm{P}_{2} \mathrm{O}_{5}$ -

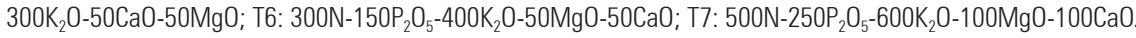

Clasificación de frutos: C6: 2,5-3,2 kg; C8: 2,1-2,5 kg; C10: 1,7-2,1 kg; C12: 1,4-1,7 kg, C14: 1,2-1,4 kg.

Tabla 7. Análisis económico de piña, cultivar MD2 ('Golden'), en función de la aplicación de abonos sintético y fertilizante orgánico. Valores en US\$.

\begin{tabular}{|c|c|c|c|c|c|c|c|}
\hline \multicolumn{8}{|c|}{ Estado de pérdidas y ganancias } \\
\hline Entradas & $\mathrm{T} 1$ & T2 & T3 & T4 & T5 & T6 & $\mathrm{T7}$ \\
\hline Ventas & $8.134,82$ & $8.132,95$ & $7.556,25$ & $14.681,52$ & $18.775,72$ & $19.894,28$ & $24.330,00$ \\
\hline Total de entradas & $8.134,82$ & $8.132,95$ & $7.556,25$ & $14.681,52$ & $18.775,72$ & $19.894,28$ & $24.330,00$ \\
\hline \multicolumn{8}{|l|}{ Salidas } \\
\hline Costo de producción & $9.494,69$ & $10.525,94$ & $10.994,69$ & $10.759,69$ & $11.136,85$ & $10.815,32$ & $11.569,63$ \\
\hline Gastos administrativos & $1.753,42$ & $1.753,42$ & 1. 753,42 & $1.753,42$ & $1.753,42$ & $1.753,42$ & $1.753,42$ \\
\hline Impuesto a la renta & - & - & - & 650,52 & $1.765,63$ & $2.197,67$ & $3.302,08$ \\
\hline Total de salidas & $11.248,21$ & $12.279,36$ & $12.748,11$ & $13.163,63$ & $14.655,90$ & $14.766,41$ & $16.625,13$ \\
\hline Utilidad & $-3.113,39$ & $-4.146,41$ & $-5.191,86$ & $1.517,89$ & $4.118,82$ & $5.127,87$ & $7.704,87$ \\
\hline Rentabilidad & $-28 \%$ & $-34 \%$ & $-41 \%$ & $12 \%$ & $28 \%$ & $35 \%$ & $46 \%$ \\
\hline
\end{tabular}

T1: Testigo; T2: Alopes Forte 25\%; T3: Alopes Forte 50\%; T4: Alopes Forte 20\%o + 150N-75P $\mathrm{O}_{5}-200 \mathrm{~K}_{2} \mathrm{O}-25 \mathrm{MgO}-25 \mathrm{CaO}$; T5: Alopes Forte 20\%o $+250 \mathrm{~N}-125 \mathrm{P}_{2} \mathrm{O}_{5}$ -

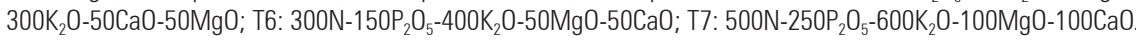

En 'Cayena Lisa', con el aumento de abono sintético a base de potasio, Teixeira et al. (2011) obtuvieron también un aumento de los ingresos brutos.

La rentabilidad fue diferente en función de las fuentes de abonos usadas (Tab. 7). Las rentabilidades más bajas fueron observadas en la producción de piña sin abono sintético, mientras que con el uso de fuentes minerales de rápida absorción, hubo mayor rentabilidad en este cultivo. Este hecho, puede estar relacionado, al incremento del peso fresco a medida que se aumentó la dosis de abono sintético aplicado.

\section{CONCLUSIONES}

Los resultados obtenidos en este ensayo demuestran que Alopes Forte, en las dosis usadas, con o sin abono sintético, no mejoró el crecimiento vegetativo ni productivo de la piña. El uso de fertilizantes sintéticos promovió un mayor crecimiento vegetativo, el aumento de la productividad y mayor calidad del fruto, los cuales trajeron consigo una mayor utilidad y rentabilidad, como fue observado en los tratamientos T6 (300N-150 $\left.\mathrm{P}_{2} \mathrm{O}_{5}-400 \mathrm{~K}_{2} \mathrm{O}-50 \mathrm{MgO}-50 \mathrm{CaO}\right)$ y T7 (300N-150 $\left.\mathrm{P}_{2} \mathrm{O}_{5}-400 \mathrm{~K}_{2} \mathrm{O}-50 \mathrm{MgO}-50 \mathrm{CaO}\right)$. 
Conflicto de intereses: el manuscrito fue preparado y revisado con la participación de los autores, quienes declaran no tener algún conflicto de interés que coloquen en riesgo la validez de los resultados aquí presentados.

\section{REFERENCIAS BIBLIOGRÁFICAS}

Almaguer, J. 2013. Fertilización nitrogenada, impactos sobre los rendimientos y el medio ambiente. Desarrollo Local Sostenible 6(16), 1-8.

Annisa, W., A. Susilawati y A. Jumberi. 2013. Response of pineapple on organic fertilizer and potassium on sulphate soil. Agroscientiae 20(1), 37-40.

Aseri, G., N. Jain, J. Panwar, A. Rao y P. Meghwal. 2008. Biofertilizer improve plant growth, fruit yield, metabolism and rhizosphere enzyme activities of pomegranate (Punica granatum L.) in Indian Thar Desert. Sci. Hortic. 117, 130-135. Doi: 10.1016/j. scienta.2008.03.014

Barbosa, J. y M. Junior. 2010. Agrostat-Sistema para análises estatísticas de ensaios agronômicos. Versão 1.0. Departamento de Ciências Exatas. Jaboticabal-SP, Brasil.

Bello, S., A. Julca, C. Echevarria y R. Cruz. 2015. Efecto del nitrógeno en la piña (Ananas comosus var. comosus L. Merr.) 'Cayena Lisa' en Chanchamayo, Perú. J. Interamer. Soc. Trop. Hortic. 56, 20-24.

Cardoso, M., R. Pegoraro, V. Maia, M. Kondo y L. Fernandes 2013. Crescimento do abacaxizeiro 'Vitoria' irrigado sob diferentes densidades populacionais, fontes e doses de nitrogênio. Rev. Bras. Frutic. 35(3), 769-781. Doi: 10.1590/S0100-29452013000300014

Carneiro, W., C. Silva, J. Muniz y T. Savian. 2013. Mineralização de nitrogênio em Latossolos adubados com resíduos orgánicos. Rev. Bras. Ciênc. Solo 37(3), 715-725. Doi: 10.1590/S0100-06832013000300018

Caetano, L., J. Ventura, A. Costa y R. Guarçoni. 2013. Efeito da adubação com nitrogênio, fósforo e potássio no desenvolvimento, na produção e na qualidade de frutos do abacaxi 'Vitoria'. Rev. Bras. Frutic. 35(3), 883-890. Doi: 10.1590/S0100-29452013000300027

Guarçoni, A. y J. Ventura. 2011. Adubação N-P-K e o desenvolvimento, produtividade e qualidade dos frutos abacaxi Gold (MD-2). Rev. Bras. Ciênc. Solo 35(4), 1367-1376. Doi: 10.1590/S0100-06832011000400031

Guitián, F. y T. Carballas. 1976. Técnicas de análisis de suelos. Ed. Pico Sacro, Santiago de Compostela, España.

Lacoeuilhe, J. 1987. Pineapple. In: P. Martin Prevel, J. Gagnard y P. Gautier (eds.). Plant analysis as a guide nutrient requirements of temperate and tropical crops. Ed. Technique and Documentation, Lavoisier, Francia.

Luna, R., K. Espinosa, R. Trávez, C. Ulloa, A. Espinoza y A. Bejarano. 2016. Respuesta de variedades de papa (Solanum tuberosum L.) a la aplicación de abonos orgánicos y fertilización química. Cienc. Agrar. 9(1), 11-16.

Ministerio de Agricultura y Riego. 2016. Boletín Estadístico de Producción Agrícola, Pecuaria y Avícola. Dirección General de Seguimiento y Evaluación de Políticas, Lima Perú.

Mujica, Y., A. Mena, A. Medina y P. Rosales. 2014. Respuestas de plantas de tomate (Solanum lycopersicum L.) a la biofertilización liquida con Glomus cubensis. Cultivos Trop. 35(2), 21-26.

Oliveira, A., M. Pereira, W. Natale, W. Nunes y C. Ledo. 2015. Qualidade do abacaxizeiro 'BRS Imperial' em função de dose de N-K. Rev. Bras. Frutic. 37(2), 497506. Doi: 10.1590/0100-2945-056/14

Omotoso, S. y E. Akinrinde. 2013. Effect of nitrogen on some growth, yield and fruit quality parameters in pineapple (Ananas comosus L. Merr.) plant at Ado-Ekiti Southwestern, Nigeria. Int. Res. J. Agric. Sci. Soil Sci. 3(1), 11-16.

Pegoraro, R., B. Souza, V. Maia, D. Silva, A. Medeiros y R. Sampaio. 2014. Macronutrients uptake, accumulation and export by irrigated 'Vitoria' pineapple plant. Rev. Bras. Ciênc. Solo 38, 896-904. Doi: 10.1590/ S0100-06832014000300021

Ramos, D., A. Terry, F. Soto, A. Cabrera, G. Martín y L. Fernández. 2016. Respuesta del cultivo de plátano a diferentes proporciones de suelo y bocashi, complementadas con fertilizante mineral en etapa de vivero. Cultivos Trop. 37(2), 165-174.

Rodríguez, R., I. Rodríguez, G. Lorente, D. López, R. Izquierdo, L. Borroto, C. Bonet, Y. Garza, C. Aragón, F. Podestá, R. Rodríguez y J. González. 2016. Efecto del déficit hídrico sobre cambios morfo-fisiológicos y bioquímicos en plantas micropropagadas de piña MD-2 en la etapa final de aclimatación. Cultivos Trop. 37, 64-73.

Schlemmer, M., A. Gitelson, J. Schepers, R. Ferguson, Y. Peng, J. Shanaban y D. Rundquist. 2013. Remote estimation of nitrogen and chlorophyll contents in maize at leaf and canopy levels. Int. J. Appl. Earth Observ. Geoinf. 25, 47-54. Doi: 10.1016/j.jag.2013.04.003

Soil Science Society of America. 1982. Methods of soil analisys. Part 2: Chemical and microbiological properties. $2^{\text {nd }}$ ed. Madison, WI, USA.

Spironello, A., J. Quaggio, L. Teixeira, P. Furlani y J. Sigrist. 2004. Pineapple yield and fruit quality affected by NPK fertilization in a tropical soil. Rev. Bras. Frutic. 26(1), 155-159. Doi: 10.1590/S0100-29452004000100041 
Teixeira, L., J. Quaggio, H. Cantarella y E. Mellis. 2011. Potassium fertilization for pineapple: Effects on plant growth and fruit yield. Rev. Bras. Frutic. 33(2), 618626. Doi: 10.1590/S0100-29452011000200035
Vilela, G., R. Pegoraro y V. Maia. 2015. Predição de produção de abacaxizeiro 'Vitoria' por meio de características fitotecnicas e nutricionais. Rev. Ciênc. Agron. 46(4), 724-732. Doi: 10.5935/1806-6690.20150059 